Original Article (short paper)

\title{
Impact on the physical activity level of adults at high and very high risk of developing type 2 diabetes mellitus: email follow-up intervention
}

\author{
Carla Giuliano de Sá Pinto Montenegro ${ }^{1,2}$, Rafael Mathias Pitta ${ }^{1} @$, Marcio Marega ${ }^{1}$, Roberta L. Rica ${ }^{3}$, \\ Luis Felipe Tubagi Polito ${ }^{\circledR}$, José Antonio Maluf de Carvalho ${ }^{4}$, Luana Queiroga ${ }^{1,2} \mathbb{D}^{-}$,

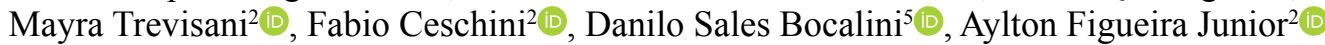 \\ ${ }^{1}$ Hospital Israelita Albert Einstein, São Paulo, SP, Brasil; ${ }^{2}$ Universidade São Judas Tadeu, São Paulo, SP, Brasil; \\ ${ }^{3}$ Departamento de Educação Física. Universidade Estacio de Sá, Vitoria, ES, Brasil; ${ }^{4}$ Hospital Beneficência \\ Portuguesa de São Paulo, São Paulo, SP, Brasil, ${ }^{5}$ Laboratorio de Fisiologia e Bioquimica Experimental do \\ Centro de Educação Física e Desporto. Universidade Federal do Espirito Santo, Vitória, ES, Brasil
}

\begin{abstract}
Aims: The present study aimed to assess the effect of a 24-week intervention on the physical activity level among adults at risk of developing type 2 diabetes mellitus (DM2). Methods: It was included 129 individuals of both sexes who underwent the Preventive Health Check-up at the Albert Einstein Israelita Hospital between January and August 2010 and who were at high and very high risk of developing DM2. The risk of developing DM2 was assessed according to the FINDRISC criteria. All participants were evaluated by the International Physical Activity Questioner (IPAQ). Subjects were followed-up for 24 weeks by a physical education professional through emails during a 6 month period. The IPAQ was sent by email after 3 and 6 months of follow-up. Statistical analysis was performed using the chi-square test with $\mathrm{p}<0.05$ and the Yule Coefficient test. Results: At baseline of the follow-up period, $80.3 \%$ of individuals failed to comply with the recommended weekly physical activity. In the end, motivational intervention by e-mail encouraged $74.2 \%$ of individuals to comply with the weeklyrecommended physical activity and only $1.5 \%$ were classified as sedentary. Conclusion: Therefore, the follow-up protocol conducted by periodic and personalized e-mails proved to be effective in promoting physical activity among people at risk of developing metabolic diseases.
\end{abstract}

Keywords: diabetes, physical activity, sedentary, diseases, lifestyle

\section{Introduction}

Physical activity (PA) practice is a key intervention in both preventing and treating cardiovascular diseases including arterial hypertension, diabetes mellitus, stroke, some types of cancer, osteoporosis, depression, and lipid profile imbalance ${ }^{1}$. PA is essential for promoting health and quality, reducing the risks of diseases, and increasing life expectancy ${ }^{1}$.

The type 2 diabetes mellitus (T2DM) is an important and growing health problem across countries worldwide, regardless of their level of development. In 1985, 30 million adults were estimated to have diabetes worldwide, increasing to 135 million in 1995, and 173 million in 2002, with projections of 366 million affected individuals by 2030; of these, two-thirds live in developing countries².

Evidence shows ${ }^{3,4}$ that sedentary lifestyle and poor quality diet are associated with a marked increase in the incidence of T2DM. Conversely, the results from the Diabetes Prevention Program (DPP $)^{4}$ showed a $58 \%$ reduction in the incidence of T2DM by promoting a healthy diet and PA practice; these interventions were more effective than drug treatment. The American College of Sports Medicine (ACSM) recommends that adults practice at least 30 minutes of moderate intensity PA five times a week to reduce the incidence of morbidity and mortality associated with non-communicable diseases ${ }^{5}$.
The advice to practice PA has been rarely applied to the specific needs of individuals, who should be encouraged to develop healthier lifestyle ${ }^{6}$. Therefore, this study aimed to assess whether following-up individuals with a high and very high Finnish Diabetes Risk Score (FINDRISC), through periodic emails, resulted in a positive intervention, encouraging changes in lifestyle and promoting regular PA.

\section{Method}

\section{Study population}

After approval from the Research Ethics Committee of Hospital Israelita Albert Einstein-SP (10456/2012), this study was carried out through the database analysis of the risk follow-up program of the outpatient clients on preventative health check-up at the Hospital Israelita Albert Einstein. The inclusion criteria applied to both men and women, of any age, with a high or very high FINDRISC and who accepted to participate in the follow-up program by signing an informed consent form. Individuals with pre-existing DM2 and Type 1 Diabetes Mellitus were excluded from this study. 


\section{Recruitment and selection}

It was included in this study 129 individuals ( $89.4 \%$ men and $10.6 \%$ women; mean age $49.27 \pm 12$ years) with a high and very high risk of developing T2DM and undergoing Preventive Health Check-up at the Albert Einstein Israelita Hospital between January and August 2010. Overall, 14 individuals refused to participate and only 66 individuals replied to the emails sent during the study period.

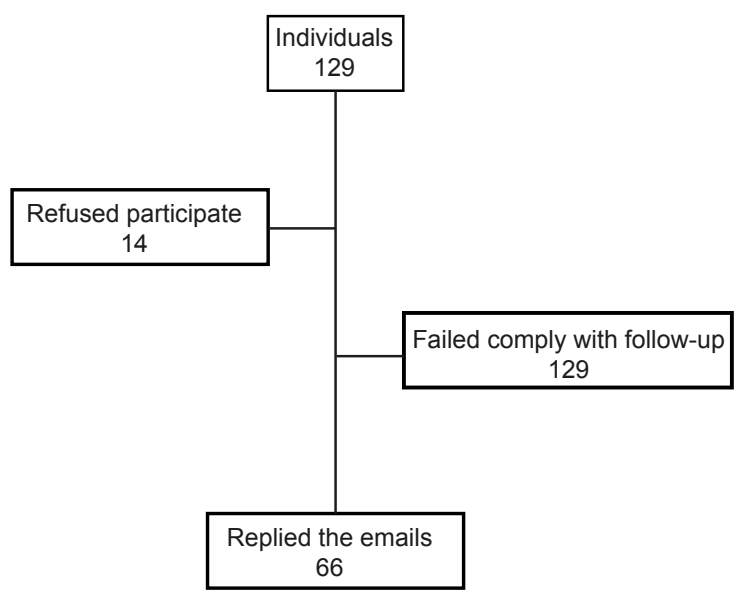

Figure 1. Recruitment

Each individual was informed about changes in nutrition needed at the time of the consultation with a physical educator, and International Physical Activity Questionnaire (IPAQ) was applied at this time. The information required for participation in the study was provided and individuals signed, of their own volition, the informed consent form to participate in the research study. Participants were then also informed about the study stages.

\section{Intervention}

Individuals underwent the Preventive Health Check-up, and on the same day, the physical education teacher sent to participants the first email, which included a table outlining the goals agreed between the individual and the healthcare professional during the consultation. Also on the same day, information about the importance of practicing PA regularly, according to the World Health Organization (WHO) criteria, was conveyed.

Personalized emails were sent weekly during the first month of follow-up, with questionnaires on PA practice and technical and motivational information. Over the second and third months, the frequency of the follow-up emails became bi-weekly. At the end of the third month, a second IPAQ was sent to each patient to assess whether changes in the level of physical activity had occurred during the first three months of follow-up.

Over the fourth, fifth and sixth months of the follow-up, emails were sent monthly, maintaining technical and motivational information.

A third IPAQ was sent to each patient at the end of the sixth month to assess whether changes in the level of PA had occurred during the first six months of follow-up.

\section{Evaluated parameters}

\section{Physical active level}

Currently, there is no evidence on the best method to measure PA. However, the IPAQ has been used as an evaluation tool in several epidemiological studies ${ }^{5,7,8}$ and provides data on PA duration, frequency, intensity, and type, thereby enabling the estimation of total energy expenditure and facilitating the classification of PA into mild, moderate, and vigorous.

During the physical education consultation, individuals were asked to complete a short version of the IPAQ, reporting the activities performed over the previous week, in order to determine their level of PA. Motivational instructions were also provided, encouraging the regular practice of PA and presenting the following message established by the Centers for Disease Control and Prevention (CDC) and the American College of Sports Medicine: "Every individual should accumulate at least 30 minutes of moderate physical activity in the most days of the week"".

To analyze the IPAQ data regarding the level of PA, we used the 2002 consensus between the Fitness Laboratory Studies Center of São Caetano do Sul (CELAFISCS) and the CDC of Atlanta, taking into account the frequency, intensity, and duration criteria of PA. Individuals were classified into four categories; 1) Very Active: individuals observing the following recommendations for PA: a) Vigorous: $\geq 5$ days/ week and $\geq 30$ minutes per session and/or b) Vigorous: $\geq 3$ days/ week and $\geq 20$ minutes per session + PA Moderate and/ or c) Walk: $\geq 5$ days/ week and $\geq 30$ minutes per session; 2) Active: observing the following recommendations for PA: a) Vigorous: $\geq 3$ days/ week and $\geq 20$ minutes per session and/or b) Moderate or Walk: $\geq 5$ days/ week and $\geq 30$ minutes per session and/ or any activity accumulated: $\geq 5$ days/ week and $\geq 150$ minutes/ week (walk + moderate +vigorous); 3 ) Insufficiently active: individuals performing PA, albeit insufficient to be classified as Active because they failed to meet the requirements regarding the frequency or duration of PA; 4) Sedentary: individuals who failed to perform continuous PA for at least 10 minutes during the week ${ }^{10,11,12,13}$.

\section{FINDRISC - Finnish Diabetes Risk Score}

This is a questionnaire used for risk stratification for diabetes and the detection of undiagnosed disease and insulin resistance. The following classification criteria are used: age, body mass index, abdominal circumference, physical activity, fruit and vegetable intake, antihypertensive medication, the presence of occasional hyperglycemia during disease or pregnancy, and family history ${ }^{14,15}$.

FINDRISC identifies individuals at high risk of diabetes who will most benefit from modifying their lifestyle ${ }^{16}$. The most relevant FINDRISC application is at the primary healthcare level, where screening strategies are necessary and widely used. 
Table 1. FINDIRISC classification.

\begin{tabular}{ccc}
\hline Score & Degree of Risk & Probability \\
\hline Up to 6 & Low & $1 \%$ \\
$7-11$ & Intermediate & $4 \%$ \\
$12-14$ & Moderate & $16 \%$ \\
$15-20$ & High & $33 \%$ \\
$>20$ & Very High & $50 \%$ \\
\hline
\end{tabular}

Risk of developing diabetes in 10 years.

\section{Statistical analysis}

Descriptive statistics were used to evaluate the frequency of the proportions of respondents. All data were analyzed using the Prism software (version 4.0, San Diego, CA, USA). The chi-square test with significance level $p<0.05$ was used for inferential analysis, and the Yule coefficient was used to identify the direction of associations.

\section{Results}

The compliance rate for remote email follow-up was $57.39 \%$ among program participants at high and very high risk for developing diabetes, according to the FINDRISC score, as $66 / 115$ individuals commencing the follow-up replied to the emails and completed the IPAQ sent. At the baseline of follow-up, $80.3 \%$ individuals failed to comply with the weekly PA recommendation and were classified as sedentary (47\%) and insufficiently active $(33.3 \%)$. The number of individuals classified as active $(47 \%)$ and very active $(12.1 \% ; \mathrm{p}<0.05)$ significantly increased after 3 months of email follow-up.

At the end of follow-up, motivational intervention by email encouraged $74.2 \%$ individuals to comply with the weekly PA recommendation, (including 50\% active and $24.2 \%$ very active participants) and only $1.5 \%$ were classified as sedentary.

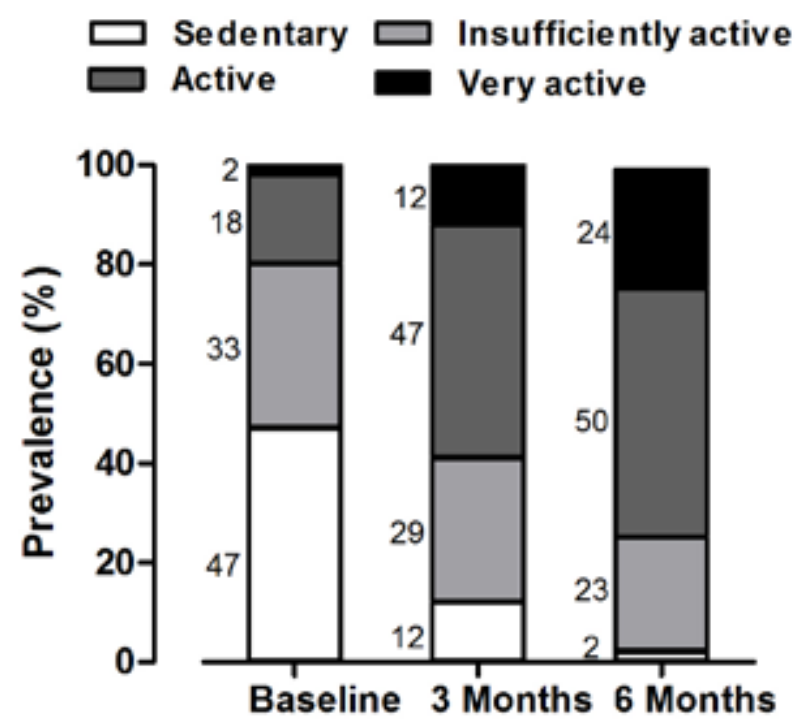

Figure 2. Physical activity level

\section{Discussion}

The fight against physical inactivity or the promotion of incentives for the regular practice of PA aims to prevent the development of cardiovascular risk factors, including T2DM. This study examined patients with high and very high FINDRISC scores by implementing an email intervention and promoting targets recommended by the WHO, specifically to perform 150 minutes of PA per week in an attempting to promote a positive change in the lifestyle. We chose to conduct follow-up by email, considering the profile of the participants. These were mostly businessmen with a high workload, who could not be easily contacted by telephone, due to work commitments, and were likely to be available online for most of the day.

Our findings are similar to another study ${ }^{3}$ on lifestyle interventions or the use of medication for T2DM prevention or onset delay. Strong evidence showed that counseling, by establishing weight loss goals and increasing the level of PA, was effective in preventing or delaying the onset of this disease ${ }^{17}$.

Programs, such as the DPP ${ }^{18}$, which aims to prevent the development of DM2, are based on dietary interventions and PA practice to counteract the excess of weight. The DPP results ${ }^{18}$ showed a $58 \%$ reduction in the incidence of DM2 cases by encouraging a healthy diet and the practice of PA, and this intervention was more effective than the use of any pharmaceutical drug, including metformin.

Decreased glucose tolerance from diabetes currently affects approximately 29 million Americans. The risk of developing diabetes over a lifetime is estimated at $36 \%$ for individuals born in $2000^{19}$. Patients with any type of diabetes have a marked reduction in life expectancy and life quality, due to specific retinal microvasculature, renal glomerulus and peripheral nerve complications, and extensive atherosclerotic macrovascular diseases affecting arteries that supply the heart, brain, and lower extremities. Other consequences include renal failure, retinopathy, neuropathy, autonomic nervous system alterations, and sexual dysfunction ${ }^{19}$.

Counseling on the practice of PA in basic health units has been scarcely applied to the specific needs of patients who should be encouraged to develop healthier living habits ${ }^{20}$.

Evidence shows that physicians who underwent specific training on PA counseling (Activity Counseling Trial) used this practice in $99 \%$ of their patients, with consultation duration varying between 3 and 6 minutes, demonstrating that this approach is affordable and easy to apply. Although the information provided adequately deals with clarifying patient doubts, these consultations are not sufficient to influence adherence to the regular practice of $\mathrm{PA}^{21,22,23}$.

A pioneering study in this area established a protocol of 3 to 5 minutes of counseling using the stages of patient behavior during clinical care and after 6 weeks. The group receiving counseling increased walking time by 37 minutes during the routine while walking time in the control group increased only by 7 minutes $^{24}$.

A systematic review conducted in different countries, including the United States and Australia, where primary healthcare is provided by pediatricians, general practitioners, 
and family physicians, showed that PA practice counseling was ineffective in promoting an increase in PA practice in patients. However, medium and long-term adherence to PA practice were obtained when the professionals explained the benefits of these changes, clarifying doubts on possible injuries, and advising on the type and intensity of exercises to be chosen ${ }^{25}$. The exchange of personalized emails with the clients followedup in this study enabled investigators to clarify any doubts regarding the parameters mentioned by Lawlor and Hanratty about PA and health ${ }^{25}$.

Telephone booster calls (Increasing Motivation for Physical Activity - IMPACT) for clarifying doubts and suggesting how to overcome barriers to the practice of regular PA was used as an adjuvant tool to lectures and leaflets for low-income women ${ }^{26}$.

The use of tools, including the Green Prescription ${ }^{27,28}$, STEP $^{29}$ and IMPACT ${ }^{25,26}$, showed a range of health benefits at patient follow-up, between 6 months and 1 year, contributing to adherence to the regular practice of physical exercise ${ }^{29}$. More positive results were achieved with verbal and written counseling (Green prescription) than with exclusively verbal counseling ${ }^{30}$.

$\mathrm{Kahn}$ et $\mathrm{al}^{31}$. conducted a systematic review to examine the effectiveness of several strategies to promote health and improve PA levels. Individual counseling based on changes in health behaviors was considered an effective method. The 18 studies selected had a positive effect on the level of PA. On average, counseling programs increased the duration of PA by $35 \%$ and energy expenditure by $64 \%$ among study participants. The number of individuals adhering to a healthier lifestyle, through PA practice also significantly increased.

Despite all the efforts made in this study, some limitations are considered. First, the level of physical activity of the study participants was not directly measured. Second, the sample size, which presented low from the application of the inclusion criteria, restricted the analysis to a specific population. Finally, the present study showed a key difference regarding the frequency of the emails, which combined with the guidance provided during the consultations, where the main tool promoting client adherence during follow-up.

\section{Conclusion}

Currently, the importance of motivational strategies encouraging individuals to become more physically active is crucial. We highlight that follow-up by repeated and personalized emails is an effective and encouraging tool for individuals at high and very high risk of developing diabetes, promoting lifestyle modifications and allowing participants to become more active by encouraging regular physical activity practices.

\section{References}

1. Francis KT. Status of the year 2000 health goals for physical activity and fitness. Phys.Ther. 1999;79:405-14.

2. Wild S, Roglic G, Green A, Sicree R, King H. Global prevalence of diabetes. Estimates for the year 2000 and projections for 2030. Diabetes Care. 2004; 27(5): 1047-53.
3. Rafferty AP, Reeves MJ, McGee HB, Pivarnik JM. Physical activity patterns among walkers and compliance with public health recommendations. Med.Sci.Sports Exerc. 2002;34:1255-61.

4. Diabetes Prevention Program Research Group. Reduction of the incidence of type 2 diabetes with life style intervention or metformin. N Engl J Med. 2002; 346(6): 393-403.

5. Pate RR, Pratt M, Blair SN, Haskell WL, Macera CA, Bouchard $\mathrm{C}$, et al. Physical activity and public health. A recommendation from the Centers for Disease Control and Prevention and the American College of Sports Medicine. JAMA. 1995;273:402-7.

6. Siqueira FV, Nahas MV, Facchini LA, Silveira DS, Piccini RX, Tomasi E, et al. Aconselhamento para a prática de atividade física como estratégia de educação à saúde. Cad. Saúde Pública. 2009; 25 (1): 203-213.

7. Rzewnicki R, Vanden Auweele Y, De BourdeaudhuijI. Addressing overreporting on the International Physical Activity Questionnaire (IPAQ) telephone survey with a population sample. PublicHealth Nutr. 2003;6:299-305.

8. Center Disease of Prevention And Control. Promoting physical activity: a best buy in public health. 2000 .

9. Pate RR, Pratt M, Blair SN, Haskell WL, Macera CA, Bouchard C, et al. Physical activity and public health. A recommendation from the Centers for Disease Control and Prevention and the American College of Sports Medicine. JAMA. 1995;273:402-7.

10. Lia V, Raele R, Conceição R, Augusto CF, Santos RD, Carvalho JAM. The Finnish Diabetes Risc Score (FINDRISC) - Avaliação do risco de desenvolvimento de diabetes mellitus tipo 2 , em dez anos, em uma população submetida a uma revisão continuada de saúde - check up. Apresentação Oral. XXX Congresso da Sociedade de Cardiologia do Estado de São Paulo.

11. Craig CL, Marshall AL, Sjostrom M, Bauman AE, Booth ML, Ainsworth BE, et al. International physical activity questionnaire: 12-country reliability and validity. Med.Sci.Sports Exerc. 2003;35:1381-95.

12. Pardini R, Matsudo SM, Araújo T, Matsudo VR, Andrade E, Braggion G. Validação do questionário internacional de nível de atividade física (IPAQ - versão 6): estudo piloto em adultos jovens brasileiros. Rev Bras Ciênc Mov. 2001;9:45-51.

13. Hallal PC, Victora CG, Wells JCK, Lima RC, ValleNJ. Comparison between short and full-length International Physical Activity Questionnaires. Journal of Physical Activity and Health. 2004; 1:227-34.

14. Lindström J, Tuomilehto J. The diabetes risk score. A practical tool to predict type 2 diabetes risk. Diabetes Care. 2003: 26: 725-731.

15. Schwarz PTH, Li J, Reiman M, Schulze J, Tuomilehto J, Lindström J. The Finnish diabetes risk score is associated with insulin resistance and progression towards type 2 diabetes. $\mathrm{J}$ Clinic Endocrinol Metabol. 2008; 94 (3): 920-926.

16. Lindström J, Peltonen M, Eriksson JG, Aunola S, Hämäläinem H, Ilane- Parikka P, Keinänem-Kinkaanniemi S, Uusitupa M, Tuomilheto J. Dats for the effectiveness of lifestyle intervention in the Finnish Diabetes Prevention Study .Diabetes Care. 2008; 31: 857-862.

17. Burnet DL, Elliott LD, Quinn MT, Plaut AJ, Schwartz MA, Chin MH. Preventing diabetes in the clinical setting. J Gen Intern Med. 2006;21(1):84-93. 
18. Diabetes Prevention Program Research Group. Reduction of the incidence of type 2 diabetes with life style intervention or metformin. N Engl J Med. 2002; 346(6): 393-403.

19. Schwarz PTH, Li J, Reiman M, Schulze J, Tuomilehto J, Lindström $\mathrm{J}$. The Finnish diabetes risk score is associated with insulin resistance and progression towards type 2 diabetes. J Clinic Endocrinol Metabol. 2008; 94 (3): 920-926.

20. Siqueira F, Facchini L, Hallal P, Piccini R, Tomasi E, Thume' E, et al. Aconselhamento para a prática de atividade física como estratégia de educação à saúde. Cad. Saúde Pública. 2009; 25 (1): 203-213.

21. Albright CL, Cohen S, Gibbons L, Miller S, Marcus B, Sallis J, et al. Incorporating physical activity advice into primary care: physician-delivered advice within the activity counseling trial. Am.J Prev.Med. 2000;18:225-34.

22. Pinto BM, Goldstein MG, DePue JD, Milan FB. Acceptability and feasibility of physician-based activity counseling. The PAL project. Am.J Prev.Med. 1998;15:95-102.

23. Harrison RA, Roberts C, Elton PJ. Does primary care referral to an exercise programme increase physical activity one year later? A randomized controlled trial. J Public Health (Oxf). 2005;27:25-32.

24. Calfas KJ, Long BJ, Sallis JF, Wooten WJ, Pratt M, Patrick K. A controlled trial of physician counseling to promote the adoption of physical activity. Prev Med. 1996;25(3):225-33.

25. Lawlor DA,.Hanratty B. The effect of physical activity advice given in routine primary care consultations: a systematic review. J Public Health Med. 2001;23:219-26.

26. Albright CL, Pruitt L, Castro C, Gonzalez A, Woo S, King AC. Modifying physical activity in a multiethnic sample of low-income women: one-year results from the IMPACT (Increasing Motivation for Physical ACTivity) project. Ann.Behav.Med. 2005;30:191-200.

27. Swinburn BA, Walter LG, Arroll B, Tilyard MW, Russell DG. The green prescription study: a randomized controlled trial of written exercise advice provided by general practitioners. Am.J Public Health. 1998;88:288-91.
28. Kerse N, Elley CR, Robinson E, Arroll B. Is physical activity counseling effective for older people? A cluster randomized, controlled trial in primary care. J Am.Geriatr.Soc. 2005;53:1951-6.

29. Petrella RJ, Koval JJ, Cunningham DA, Paterson DH. Can primary care doctors prescribe exercise to improve fitness? The Step Test Exercise Prescription (STEP) project. Am.J Prev.Med. 2003;24:316-22.

30. Swinburn BA, Walter LG, Arroll B, Tilyard MW, Russell DG. The green prescription study: a randomized controlled trial of written exercise advice provided by general practitioners. Am.J Public Health. 1998;88:288-91.

31. Kahn EB, Ramsey LT, Brownson RC, Heath GW, Howze EH, Powell $\mathrm{KE}$, et al. The effectiveness of interventions to increase physical activity. A systematic review. Am J Prev Med. 2002;22 (4 Suppl):73-107.

\section{Acknowledgements}

We want to thank the scholarships provided by FAPES (590/19 - no. $84417625 / 2018$ ) and the support of the CAPES and CNPQ.

\section{Corresponding author}

Danilo Sales Bocalini

Universidade Federal do Espírito Santo, Goiabeiras, Vitoria, Brazil

Email: bocaliniht@hotmail.com

Manuscript received on July 25, 2018

Manuscript accepted on October 22, 2018

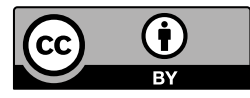

Motriz. The Journal of Physical Education. UNESP. Rio Claro, SP, Brazil - eISSN: 1980-6574 - under a license Creative Commons - Version 4.0 